\title{
Cuando las políticas sociales cuentan con los actores sociales implicados en la construcción de subjetividades y derechos
}

\section{When social policies are counting with the social actors involved in the construction of subjectivities and rights}

En los artículos de este número se plasma la reflexión sobre el Estado de bienestar, las políticas sociales que confieren derechos sociales a los usuarios y las oportunidades o limitaciones de los espacios para los colectivos vulnerables, ya sea por hallarse en situación de pobreza o por desarrollar su vida en un espacio concebido con criterios que se han visto superados por los cambios en la ampliación de derechos ciudadanos y en el uso de las tecnologías de la comunicación. Con la atención puesta en las personas que habitan en las ciudades y los barrios, y en las relaciones que mantienen con la Administración Pública y los profesionales de la intervención social, las articulistas llevan sus reflexiones acerca de las políticas sociales y la intervención social al terreno internacional y español. De las diecinueve articulistas solo cinco son varones; quince autoras son españolas y cuatro latinoamericanas/os; dos de los artículos ofrecen también su versión en inglés. Tres artículos ofrecen una perspectiva internacional: uno con una comparativa entre modelos sociales europeos y dos de casos latinoamericanos; los demás son ensayos y análisis de casos de interés para el Trabajo Social.

Aquellos con la mirada puesta en la construcción del conocimiento y de las relaciones entre los usuarios y las Administraciones públicas, como sujetos de conocimiento y destinatarios de las prestaciones de servicios y prestaciones económicas que erogan, es el hilo conductor del debate entre distintos $\mathrm{y}$, a menudo, contrapuestos intereses, como se analiza en los artículos siguientes.

Con carácter empírico y una perspectiva comparada de dos modelos de Estado de bienestar europeos, la construcción de las relaciones entre los sectores proveedores de servicios y prestaciones del Estado de bienestar y su transformación en el contexto de la glo- balización es el argumento de Las personas mayores en el Estado de bienestar: las politicas sociales en Alemania y España, de Amezcua-Aguilar, Alberich-Nistal y Sotomayor Morales. Estos autores definen los fenómenos y objetivos del Estado social, y las corrientes que, con perspectivas contrapuestas, ponen en tela de juicio las políticas de servicios sociales. Se toman como referencia las crisis sobrevenidas -1973 y 2008- para cuestionar normas y políticas sociales de los dos modelos analizados: el corporativo-subsidiario alemán y el mediterráneo-familiar español; tema de fondo económico y financiero que arroja algunas de las diferencias y analogías entre los modelos sociales de ambos países, y que no son pocas, dicen los autores. Entre ellas, el consenso en relación a la respuesta positiva del Estado de bienestar para las personas vulneradas por el sistema, pero también en su limitado alcance que solo llega a las personas pobres, y que no ha evitado reducir, incluso en la coyuntura actual, la protección social pública a las necesidades de estas personas mayores. El fenómeno de limitación en la oferta de recursos ha activado, por un lado, a la sociedad civil como partner del sector público; y por el otro, a la sumisión del ciudadano potencial usuario y el Estado social al irremediable protagonismo del mercado, en la provisión de la atención social privada o concertada, que acaba dictando las reglas del bienestar, mientras la sociedad y sus individuos aceptan la retirada del sector público como una deriva inexorable. El Estado de bienestar declara abiertamente su incapacidad para ofrecer otros recursos que no sean los de mejorar la situación económica y social de los colectivos en riesgo de exclusión social, pero no su voluntad de poner en marcha políticas sociales alternativas que reduzcan las desigualdades e inseguridades que provoca la globali- 
zación económica. A pesar de que las personas entrevistadas en esta investigación reconocen que las políticas fiscales y las transferencias monetarias han sido importantes, se muestran impotentes a frenar la irrupción de la economía globalizadora. No queda más remedio, pues, que la hibridación de las lógicas de los actores sociales que impulsaron el Estado de bienestar social. Por un lado, corresponsabilizar al Tercer sector, que se erige ahora como el partner privado del Estado de bienestar en gestión y financiación de los servicios sociales, dando lugar al modelo mixto de bienestar social que se está consolidando en Europa. De este modo, el voluntariado se convierte en un pilar demasiado importante en la toma de decisiones políticas, delegándole responsabilidades que modifican su propia naturaleza. Por este sistema de gestión del Tercer sector y por su dependencia financiera es por lo que pierde su independencia, "aligera" sus principios y su posición contractual, fomentando con ello su clientelismo. Por el otro, el único de los actores que atienden a las necesidades sociales que no se hibrida su lógica, sino que mantiene su coherencia es el mercado, que ahora irrumpe con fuerza en el campo de lo social, y presiona para pervertir los principios del resto de los actores sociales, que abandonan lo que en origen había sido su lógica esencial.

El ensayo de Kleba Lisboa y Nascimento de Oliveira, El saber surge de la práctica: por un Trabajo Social bajo una perspectiva feminista, trata de las características de las políticas universitarias en Brasil y de su resistencia a incorporar marcos teóricos, como los estudios feministas, de género e interseccionales, en los planes de estudio universitarios. El predominio del pensamiento marxista en la Universidad, centrado en los análisis de clase y críticos con otras miradas, como la de género, ha obstaculizado una reflexión teórica dialéctica alternativa, refieren las autoras. No obstante la academia y la profesión del Trabajo Social brasileño sigue en la lucha por otorgar un estatus académico y de igualdad a los saberes que producen las mujeres, con ellas y sobre ellas, considerándolo fundamental por la profundidad que aporta el abordaje de las desigualdades de género y sus interseccionalidades al conocimiento científico. Las publicaciones recientes sobre políticas de igualdad-dicen las autoras-ya han incorporado la interseccionalidad en las políticas públicas, y han tratado de evitar la superposición de categorías de diferenciación, mediante su análisis como elementos complementarios, profundamente imbricados y con efectos diferentes, según el contexto. Aun así, no han concedido el suficiente valor a la categoría "experiencia" de las mujeres. Lo resaltan tres de las principales tendencias propuestas por las autoras: la teoría del punto de vista (Standpoint); el empirismo feminista; y el postmodernismo feminista, para analizar el fenómeno detenidamente. El que no hayan encontrado eco suficiente fundamenta su rechazo a reconocer otros saberes alternativos o complementarios para analizar las desigualdades sociales. Sin embargo, la claridad teórica que orienta el ejercicio profesional de los trabajadores y trabajadoras sociales es producto de la complejidad y el entrecruzamiento de las cuestiones sociales, con unos marcos teóricos más amplios como los que aportan las autoras del artículo y tratan de impulsar en el artículo el cambio de actitud de la academia brasileña.

El enfoque feminista, en la perspectiva relacional, es la inquietud que plantea en su artículo Ríos Campos. En el ensayo, Aportaciones de las teorías relacionales y feministas al Trabajo Social, analiza la interacción del individuo con el medio social, como un elemento en el que las teorías feministas y el enfoque relacional enriquecen el análisis en numerosos aspectos, tanto en los individuales como en aquellos compartidos entre sujetos. Este enfoque con una perspectiva de Trabajo Social feminista aúna ambos marcos por lo que tienen en común, y da a la intervención social una mirada interdisciplinar. El camino que emprenden en común, hacia la transformación social y la eliminación de las desigualdades con la participación de los usuarios, constituye el fundamento compartido de las teorías y perspectivas feministas y la disciplina y la práctica del Trabajo Social. El enfoque feminista analiza los problemas sociales no tanto bajo la perspectiva de clase, sino con la del impacto específico identitario que tienen las desigualdades sociales sobre las mujeres, y por ello la autora del artículo considera más apropiada porque, de no tenerlo presente, deriva tergiversar lo que es "pretendidamente 'universalista' [pero] que, realmente termina siendo patriarcal, racista, clasista, heterocéntrica, etc.". Por el contrario, razona la autora, que las mujeres encuentran sometidas a otras discriminaciones más allá de la de clase, que son múltiples y por este motivo son sujetos de múltiples exclusiones, por lo que es necesario que el Trabajo Social incorpore las miradas analíticas diver- 
sas del feminismo, para abordar esas desigualdades, de manera simultánea o interseccional, deteniéndose tanto en los privilegios como en las opresiones, señala la autora.

Las argumentaciones de los dos artículos siguientes focalizan la función de las políticas sociales en la construcción de nuevas subjetividades y en la conformación de los derechos subjetivos de los destinatarios. Ambos artículos se sitúan en un contexto internacional argentino e invitan a la reflexión acerca de la aplicación de las políticas de transferencias monetarias, cuya función es atribuir derechos a otros sujetos anteriormente desprotegidos. Con estos sujetos, empobrecidos y además jóvenes, se plantea la posibilidad de que obtengan o reclamen aquellos derechos que les son propios. Se fija la atención en la diversidad de intereses -a menudo en conflicto- entre los actores implicados en la transferencia de recursos monetarios, y en el enriquecimiento que supondría que la actuación política se adecuara a las demandas de los sujetos interesados, porque ello contribuiría también a la transformación de las mismas instituciones.

Hornes y Maglioni, en su artículo Accesibilidad y politicas sociales en la Argentina reciente: una interpretación desde los saberes expertos en programas de transferencias monetarias (publicado también en inglés en este número), centran el interés en las políticas sociales argentinas de la etapa kichneriana. Iniciaron en 2002, han ido ampliando no solo el número de perceptores de las prestaciones económicas, sino han agilizado también los requisitos de acceso a ellas, se trata de políticas sociales que se interrumpen, en 2015 con la llegada de Macri a la presidencia. Diferentes expertos en políticas sociales de los organismos nacionales y supranacionales de otros países latinoamericanos (México y Brasil, entre otros), ponen en común sus conocimientos para definir qué/ necesidades y qué criterios se han de fijar para redistribuir las ayudas, y para analizar las posibilidades de ampliarlas, teniendo presente el momento -de recesión o de recuperación económica- en el que se halle el país. Según los autores, la oportunidad de haber abierto a nuevos y diversos titulares ha focalizado mejor las necesidades y ha ido más allá de la contención social o la inserción laboral, funciones que tradicionalmente se atribuyen a las políticas de bienestar social, sino que se han contemplado las transferencias monetarias: "como formas de restauración de derechos a distintos grupos etarios y sociales"; y han brindado otras soluciones al fenómeno de la pobreza y la diversidad de sujetos que cargan con ella. Sin embargo, insisten los autores, una mayor -y más ágil- disponibilidad de prestaciones económicas no debe prescindir de una oferta de otros servicios sociales de calado -como los de salud y educación- y el consiguiente mejor acceso a estos servicios, si se quiere que tenga lugar tanto la transformación de los sujetos como la de las instituciones y quienes se hacen eco de ellas: los profesionales de la intervención social.

También en perspectiva latinoamericana, el artículo Complejidades, responsabilidades e invenciones: construcciones de subjetividades y de derechos en el encuentro entre instituciones y jóvenes de sectores populares (con una versión también en inglés), de Arias y Di Leo aborda el reflejo de las políticas sociales en aquellas organizaciones "particulares" y en "las formas socialmente construidas" con las que los jóvenes empobrecidos urbanos organizan y proyectan sus vidas para afrontar la exclusión, amenaza y realidad. Estos jóvenes de las áreas marginales urbanas cuentan con un "patrimonio de desigualdades" tal que condensa y superpone diversos elementos: la exclusión del acceso al mercado laboral y el abandono de la protección social pública que les confina a un espacio limitado, el barrio -en el que se hallan encerrados- que por un lado, incrementa aún más su vulnerabilidad, pero que, por el otro, se configura como una institución que les apoya y compensa ese déficit de recursos públicos y soportes afectivos o simbólicos, ayudando a afrontar la fragilidad de esta juventud. A veces, algunas instituciones públicas -las educativas sobre todo- $y$ otras organizaciones sociales son capaces de crear un capital social intergeneracional en y con estas "instituciones diferentes"; un valor identitario que se ve reforzado por la procedencia de clase y de género. Los autores señalan que los "marcos referenciales" barriales que construyen las identidades individuales y la perspectiva transformadora de estas instituciones, impulsan a los jóvenes a su construcción como sujetos de derecho, a desarrollar sus capacidades como sujetos activos y transformadores en este proceso de construcción de derechos; pero, que por otro lado, también las instituciones están más interesadas en ellos, pero más en "activarlos" que en transformarlos. No obstante, la tarea institucional es compleja; por un lado, tratar de lograr que el 
otro "quiera" transformarse es un gran desafío para las instituciones, y por el otro es también un espacio de limitación. El método para articular las instituciones con los sujetos-objeto de la intervención requiere definir bien el problema y las personas que lo cargan, teniendo claro que ni las personas usuarias son el problema ni tampoco las instituciones exclusivamente, sino que unas y otras son parte del mismo y de su solución. De ahí que para el análisis de la situación, en el diagnóstico el trabajador social ha de preguntarse en qué institución está, con qué recursos cuenta y quién lo financia; de ese modo, podrá aclarar desde el principio, que su intervención no es unidireccional sino dialogada entre profesionales y usuarios, pero en la que concurren además otros elementos; unos más visibles que otros: los institucionales (recursos, condiciones, orientaciones, documentación, etc.) además de las prestaciones que ofrecen al usuario, el posicionamiento ideológico acerca de los recursos y la elaboración de estrategias, entre otros. Así se comprende mejor la dimensión política subyacente a su trabajo, dicen los autores.

La apuesta por la metodología participativa es también uno de los elementos que se resalta en la investigación del artículo, El derecho de participación en las políticas sociales: el proceso participativo para la elaboración de la Ley valenciana de Servicios Sociales, de Caravantes López de Lerma. Poner al frente del equipo directivo a los profesionales de Trabajo Social de reconocida experiencia en el ámbito de los servicios sociales, describe no solo el proceso de elaboración y profundización de las aportaciones de los diferentes actores sociales, sino el resultado de la publicación, en mayo de 2019, de la nueva Ley 3/2019, de Servicios Sociales Inclusivos, tercera ley $(1989,1997$ y 2019). La ciudadanía, los agentes sociales, los recursos técnicos y la Administración Pública son los elementos esenciales y protagonistas del proceso de debate y se su resultado. Dividido en cuatro etapas, en las que se incluye a los componentes del proceso participativo - de octubre de 2015 a febrero de 2019- y se profundiza en el diseño de los mecanismos e instrumentos de participación y en los resultados que se van obteniendo en cada una de ellas. Enriquece el procedimiento, el haber contado con la colaboración de la Federación Valenciana de Municipios y Provincias -los 542 municipios de la Comunitat Valenciana, de Castellón, Valencia y Alicante- así como con la de sin- dicatos, colegios y asociaciones profesionales, universidades públicas valencianas, empresas y entidades que desarrollan su actuación en el ámbito de los servicios sociales. Ha supuesto -según la autora- poder considerar una iniciativa común, compartida y plural la elaboración de esta nueva Ley de servicios sociales valenciana que se convierte, por ello, en un proyecto de la sociedad valenciana, y crea un precedente para llevar a cabo otras iniciativas políticas de carácter social. Se trata, por lo tanto, de una la respuesta incluyente del sistema político-administrativo y ciudadano a la situación crítica en la que se encuentran los servicios sociales en la Comunitat Valenciana.

De la creación de contextos de colaboración y no solo como espacios de contención o de reconocimiento mutuo, sino como algo que es más importante para recuperar a los profesionales reflexivos con quienes "repensar la acción práctica en su dimensión teórica, técnica y ética", trata el tema y el contenido del ensayo, El trabajo en red colaborativo: desafios y posibilidades. González-Abad y Rodríguez Rodríguez ofrecen una reflexión sobre el trabajo social en red, los desafíos, las posibilidades y estrategias para llevar a cabo la colaboración entre quienes la construyen. Describen los distintos niveles de colaboración y las dificultades principales, aclaran lo que entienden por cooperación, coordinación y colaboración y para profundizar posteriormente en las relaciones interinstitucionales, interprofesionales $\mathrm{y}$ aquellas que se fraguan entre profesionales y usuarios. Lejos de ser fácil, el trabajo en red se topa con diversas dificultades no solo en el marco de la colaboración, sino en el de las relaciones interpersonales, unas de carácter macro social, otras relacionales y otras de marco micro social. Entre las primeras, permanece vigente un paradigma positivista simplista que dificulta el paso de la visión naturalizada como única y real a la realidad compleja que se construye "con base en la vivencia del sujeto, la mirada de los profesionales y la escucha que entre ellos pueda emerger". El hecho de que existan culturas organizativas, contrapuestas no solo en relación a la toma de decisiones, sino al modo de entender los diagnósticos y planificar los encuentros, así como las motivaciones que justifican la participación en el trabajo en red, se complica con la escasez de recursos y la necesidad de financiación que juegan un papel importante en su buen funcionamiento. Las dificultades en las relaciones entre profesiona- 
les y agencias derivan tanto de la confusión en la comunicación entre mensajes y relaciones de poder entre actores, como de la réplica en los servicios de los patrones relacionales de los usuarios. Asimismo las autoras señalan los riesgos microsociales a la hora de trabajar en una red colaborativa, como son: el temor a perder una parte o toda la misma "esencia de uno (...) así como el control sobre áreas y asuntos considerados propios", sin ver las ventajas que aporta la multiprofesionalidad en el trabajo en red, en términos de enriquecimiento individual y de la propia red; máxime frente a la impotencia percibida cuando no se puede solucionar el problema, que suele llevar a poner "a la defensiva a la agencia y al profesional". Tampoco se ha de olvidar que la presencia de los conflictos no debe eludirlo la red ni tratar de minimizar su alcance o su contenido, menos aún cuanto el conflicto se presenta entre organizaciones y profesionales, que suele derivar de la interdependencia funcional y la escasez de recursos. Por el contrario, "Normalizar el conflicto es el primer paso, para poder abordarlo", dicen las autoras. En fin, González-Abad y Rodríguez Rodríguez cierran su reflexión, como lo hacen a lo largo del artículo, preguntándose ¿cómo se trabaja en red colaborativa? Y responden con cinco propuestas claras, no por ello menos complejas: aceptar la incertidumbre como elemento habitual; considerar al "otro" como un sujeto competente; salirse de la posición de omnipotencia y aceptar la concurrencia de otros en el abordaje de la necesidad social; desplazar el monólogo al diálogo polifónico que, sin duda, aporta mayor comprensión; e incorporar en este diálogo y en la acción a los usuarios y sus redes naturales, como parte del trabajo en red. La reflexión acerca de que es necesario escuchar, tal vez más que de hablar, y reconocer al "otro", incluyendo al usuario, como capaz y necesario, es una de las conclusiones de cierre del artículo.

Los dos artículos que siguen abordan dos temas, distintos en apariencia pero unidos por el discurso de los sujetos y por la apropiación de las nuevas (o no tan nuevas) tecnologías. Persiguen dar visibilidad a aquellos sujetos de derecho que analizan los autores: por una parte, los padres que son objeto de maltrato por sus hijos y, por el otro, las personas en situación de discapacidad sensorial, excluidas por sus capacidades sensoriales por ser diferentes, por diversas condiciones corporales, de la mayor parte de la población, pero que no ello deben ser negados también sus derechos de ciudadanía. Los audiovisuales, por un lado, y el uso de las tecnologías que externalizan los sentidos en el contexto urbano, colocan al espacio urbano como el campo de interacción e interdependencias con los sujetos, y son elementos que pueden articular el discurso y las representaciones de ambos artículos.

En el artículo, Análisis discursivo de la violencia filioparental en diferentes producciones audiovisuales, De la Rosa y las dos autoras Palma García analizan este fenómeno, en el contexto actual. El análisis del discurso de algunas producciones audiovisuales (repertorios) que representan esta realidad, hacen constatar a las autoras un cambio en lo que se considera la violencia filioparental, pasando de la violencia tradicional "reactiva" ante una agresión o un abuso de poder de los progenitores a otra violencia "gratuita", en familias aparentemente "normalizadas" de todas las clases sociales en las que no constaba que hubiera habido previamente ningún tipo de problema en este sentido. Definida como un maltrato reiterado, constante y cíclico a los padres para hacerles daño y conseguir cuotas de "poder, control y dominio sobre sus víctimas para conseguir lo que desean", el artículo aborda el fenómeno con un marco teórico prolijo - a teoría del aprendizaje social, las teorías instintivas y psicosociales y sus respectivos modelo - para analizar un problema que implica diversos ámbitos: legislativo, profesional y de la comunicación. Su finalidad es preventiva y sacar del ámbito privado para darle visibilidad pública, apoyándose en la sociedad de la información y en cómo se construye la violencia filioparental en los medios de comunicación, aportando mediante algunos documentales, las estrategias discursivas que usan los actores para legitimarlo o deslegitimarlo, mediante las opiniones de los distintos agentes implicados en estos repertorios (2008-2015). Señalan las autoras, por un lado, que la función de los medios de comunicación social tienen una orientación preventora y educadora y, por el otro, recomiendan que la intervención social en estos casos sea integral, con todos los miembros de la familia en cuestión. En el artículo queda claro que los mass media, por un lado evidencian la realidad del maltrato filioparental, y por el otro exigen el compromiso para erradicarlo y una responsabilidad de todas las esferas sociales implicadas en su prevención y atención a este fenómeno. 
En el artículo, Multicorporalidad frente a ocularcentrismo: de la ciudad ojo-individuo a la ciudad sensorial-participada, Moya Santander y Bergua Amores analizan, con Ruiz Pérez, un proyecto original de fin de carrera en Ingeniería de diseño industrial. Afrontan el uso de las tecnologías como formas de externalización de los sentidos en la ciudad, como el terreno de interacción e interdependencias con los sujetos. Este artículo cuestiona los límites de los paradigmas modernos con los que se han construido las ciudades -el visualcéntrico y tactilocéntrico- en que se ha pasado del sujeto oral al alfabetizado, como lectores de textos tipográficos o analfabetos, pero que han excluido las perspectivas sensoriales de aquellos sujetos que difieren por diversas condiciones corporales. La metodología participativa con las personas que intervienen en la acción -incluidos los vecinos- han podido experimentar que los sentidos, externalizados en formas de tecnologías, permean los espacios de maneras diversas, y dan lugar a una serie amplia de experiencias de los cuerpos en el entorno analizado. Las necesidad de transformar el paradigma modernizador de la ciudad mediante la acción social comunitaria - a partir de la teoría de los tres círculos, denominados "núcleo", "colaboradores parciales" e "informativo"- no solo puede transformar la sociedad sino también cuestionar las intervenciones asistenciales que dan respuesta las necesidades de las personas en situación de discapacidad. Por ello han de ir más allá de la mera intervención puntual, sino que tienen que profundizar en cuestiones estructurales, como son las relaciones de poder que prescinden o ignoran los conocimientos y las capacidades de los sujetos, de las personas con condiciones corporales diversas. Moya Santander, Bergua Amores y Ruiz Pérez reflejan ambas perspectivas en este original artículo, y lo hacen aplicando el enfoque participativo e interdisciplinar de la metodología Journey Mapping con lo que exploran las posibilidades que brinda la ciudad a quienes habitan en ella. Además, actualmente, una oportunidad para el cuerpo que no se debe desaprovechar es la de las nuevas tecnologías que han "desplazado a la vista de su posición privilegiada y ha puesto en un primer plano la corporalidad ampliamente entendida, abriéndose así un mundo de sentidos interrelacionados"; muy a pesar de la teoría arquitectónica, que ha tendido a considerar el espacio como un objeto disociado del sujeto, y que sin embargo este espacio se revela como interactivo e interrelacionado para los sujetos con otras potencias sensoriales. La técnica de resistencia que utilizan los autores, con la que asignan valores positivos a rasgos tradicionalmente considerados como negativos -la reapropiación de la palabra crip ("tullido", "tarado", etc.) por las personas en situación de discapacidad sensorial- se realiza para subvertir -dicen los autores- la estructura jerárquica de poder, basada en la relación de poder dominante-subalterno, y le confieren otro significado positivo que refleja un cuestionamiento más del orden establecido.

En los artículos que integran este número, las políticas sociales arrojan unos resultados tanto a escala macro como micro, que contribuyen a ampliar los espacios de la participación social. No solo como consecuencia del repliegue del sistema de protección público, o por la falta de voluntad política de afrontar los riesgos estructurales, sino por propia iniciativa de los sujetos vulnerables interesados en acceder a sus derechos ciudadanos, se ha avanzado en el terreno normativo, aunque sea tímidamente, y el conjunto de los actores implicados tienen mayor conciencia de su responsabilidad para afrontar, participadamente, los retos presentes y futuros generadores de más desigualdades e inseguridades sociales -aquellas que provocan unas políticas económicas y financieras globalizadoras de cuyos beneficios están excluidospara generar un capital social y unas relaciones de autocuidado y heterocuidado incluyentes con todos los actores implicados en la acción social.

Teresa García Giráldez 